Aus der dermatologischen Klinik der Kgl. Universität Parma.

(Vorstand: Prof, V. Mibelli.)

\title{
Über die Dermatitis exfoliativa neonatorum (Ritter).
}

\author{
Von \\ Dr. G. B. Dalla Favera, \\ Assistent.
}

(Hiezu Taf. VI.)

Der Fall, welcher mir Veranlassung zu dieser Arbeit bot, hat sich im Winter 1909 in der dermatologischen Klinik zu Parma vorgestellt. Auf Grund des objektiven klinischen Bildes stellte Prof. Mibelli die Diagnose: Dermatitis exfoliativa neonatorum. Ich konnte ron diesem Fall die folgende klinische Gescbichte zusammenstellen:

G. N. aus Parma, 25 Tage alt, wurde am 19./II. 1909 in die Klinik aufgenommen und starb an demselben Tage um 8 Uhr Abends.

Pat. ist das erste Kind von sehr gesunden Eltern, und wurde, nach einer ganz normalen Schwangerschaft, zur rechten Zeit geboren; Wochenbett der Mutter verlief normal. Pat. wies bei der Geburt und wälrend der ersten Tage nach derselben keine Veränderungen der Haut auf.

Die ersten Hautveränderungen traten ungefähr 10 Tage nach der Geburt auf, und zwar in Form von einer lebhaften, auf den Wangen und besonders in der Umgebung des Mundes lokalisierter Rötung. Diese erythematöse Verfärbung breitete sich langsam über die Stirn, die Schläfen, die Ohrmuscheln aus, so daß sie schlieBlich das ganze Gesicht bedekte. Dieser Zustand dauerte ungefähr eine Woche; erst seit zwei Tagen hatte sich die Rötung über die ganze Haut des Körpers ausgebreitet und es waren die schweren Hautveränderungen aufgetreten, welche ich weiter

1) Ins Deutsche übertragen von Dr. med. K. Rühl, Torino. 
unten beschreiben werde. Ebenso waren seit zwei Tagen, zu gleicher Zeit mit der Verschlimmerung der Dermatose, Magendarmstörungen eingetreten: Appetitlosigkeit, Aufstoßen, grünliche Stublentleerungen. Das Kind wurde dabei sehr unruhig. Am Tage vor der Aufnahme in die Klinik ist die Bindehaut beider Augen stark angeschwollen, so daB jede aktive und passive Bewegung der Augenlider unmöglich wurde. Auch haben sich die Nasenwege verstopft, so daß das Kind nicht mehr an der Brust saugen konnte.

Status praesens (19./II. 1909). Ziemlich guter Ernährungszustand; Körpergewicht $4700 \mathrm{~g}$. Die Haut weist in ihrer Gesamtheit eine intensive diffuse und fast gleichmäßige rote Farbe auf, welche im Gesicht und auf dem Rumpf einen dunkleren, fast violetten Ton annimmt. Die Haut fühlt sich heib an und ist angeschwollen - was man merkt, wenn man sie in Falten hochziebt - ; die normalen Hautfalten sind in der Weichengegend und an den Gelenken ausgesprochener als gewöhnlich. Die ganze Hautoberfläche befindet sich in einem Exfoliations. zustande. Diese Abblätterung geschieht nur an einigen Stellen in Form von feinen, ziemlich trockenen und leicht loslösbaren epidermischen Schuppen; in den übrigen Zonen geschieht die Exfoliation in Form von breiten Fetzen, welche aus den oberen Epidermisschichten gebildet sind. Diese sind von den unterliegenden Schichten abgetrennt und über denselben beweglich; sie haften aber, dank ihrer Fenchtigkeit, an denselben bis zu einem gewissen Grade an und bedecken sio in Form einer opaken, gekräuselten und runzligen Follikel, welche die intensive Rötang der unterliegenden Oberfläche etwas abschwächt. Wenn man einige dieser Hautfetzen entfernt, kommt eine hellrote, glänzende, trockene oder wenig feuchte Fläche zum Vorschein. Wenn man mit dem Finger über Hautzonen streicht, in welchen die Epidermis auf den ersten Blick kompakt erscheint, so erzielt man leicht dasselbe Resultat, indem man die oberen Epidermisschichten über den unteren verschiebt und dann leicht loslösen kann.

An mehreren Stellen, besonders auf dem Rücken und auf den Streckflächen der Arme, ist die Exsudation durch die Epidermis stärker and die Exfoliation geschieht, ohne die beschriebenen Charaktere zu verlieren, in Form von dickeren, gelblichen, krustenähnlichen Schuppen. An noch anderen Steilen, an welchen die Veränderungen der Epidermis ihren böchsten Grad erreicht haben, zeigen die ziemlich dicken und gelblich-braunen krustenähnlichen Schuppen, bzw. schuppenähnlichen Krusten tiefe, rhagadenähnliche Spalten, durch welche man eine blutige Fläche sehen kann. Diese Veränderungen, welehe der vollständigen Epidermiserosion entsprechen, findet man besonders an den Mundwinkeln, an den Furchen zwischen Nase und Backe, an den Augenliderfugen, auf dem Kinn, und auch hier und da auch auf dem Rumpf zerstreut.

Die spärlichen Haare sind besonders anf dem Hinterhaupt durch diffuse, feuchte krustenförmige Gebilde miteinander verklebt. Die Handteller und Fußsohlen weisen keine Veränderung auf, abgesehen von einer mittelstarken erythematösen Verfärbung. 
Das Kind weist eine starke Chemosis anf mit leichter Augenlider. ektropion und Tränenfluß. Infolge der Anschwellung der Bindehaut gelingt es nur mit Schwierigkeit, die Hornhaut bloßzulegen; dieselbe ist intakt.

Die Mundschleimhaut weist nur eine diffuse erythematöse Rötung auf. Die Stimme ist nicht verändert.

Der Zustand des Kindes zeigte am Tage der Aufnahme in die Klinik gegen Abend eine rasche Verschlimmerung. Temperatur $38^{\circ} 8^{\circ} \mathrm{C}$; wiederholtes und bäufiges Erbrechen. Das Kind starb plötzlich nach einer starken Hämatemesis.

Aus dem Sektionsbefunde (21./II. 1909) erwähne ich nur diejenigen Punkte, welche von einigem Interesse sein können:

Kehlkopf, Laftröhre und Bronchien sind kongestioniert und weisen eine leichte hämorrhagische Imbibitionsverfärbung anf.

L ungen sind frei von Verwachsungen, kongestioniert, lufthaltig; auf ihrer freien Oberfläche sind äußerst zahlreiche, punktförmige und zusammenfließiende subpieurale Hämorrhagien sichtbar.

Herz ist normal. Ebenso Leber, Pankreas und Nieren.

Die Speiseröhre ist leicht kongestioniert und enthält kleine frische Blutgerinnsel. Solche findet man auch im Magen, dessen Schleimhaut gleichmäßig kongestioniert ist und zahlreiche Kapillarbämorrhagien zeigt. Im Darm ist, von den letzten Abschnitten des Kolons bis zum Coecum die Schleimhaut mit Lymphknoten übersät, welche auf ihrer leicht hyperämischen Oberfläche in Form von stecknadelkopfgroßen, an der Peripherie stark kongestionierten und einen zentralen weißlichen Punkt aufweisenden Erbabenheiten deutlich hervortreten. In einigen der Lymphknoten ist im Zentrum eine kleine graurote Ulzeration sichtbar.

Anatomische Diagnose: "follikuläre Enterocolitis, Dermatitis exfoliativa".

Sofort nach dem Tode des Kindes setzte ich mit dem Blute Kulturen auf Agarbouillon und auf Peptonbouillon an. Zur Zeit der Obduktion setzte ich weitere Kulturen mit dem Herz- und Milzblut an. In fast allen besäten Röhrchen entwickelten sich üppige Kolonien eines dem Gram widerstehenden Staphylococcus aureus. Einige Röhrchen blieben steril.

Der von mir gezüchtete Mikroorganismus befand sich im Zustande absoluter Reinheit, wie ich mich durch Plattenaussaat überzeugen konnte.

$1 \mathrm{~cm}^{3}$ von Bouillonkultur dieses Staphylococcus tötete ein $1 \cdot 400 \mathrm{~kg}$ schweres Kaninchen, dem ich es in die Ohrenrandvene eingespritzt hatte, in 24 Stunden. Aus dem Herzblut und aus der Milz des Kaninchens konnte ich wieder den eingeimpften Staphylococcus züchten.

Zwecks histologischer Untersuchungen entnahm ich der Leiche sofort nach dem Tode Hautstücke von der äußeren Oberfläche der Arme, von der vorderen Schenkeloberfläche und vom Rücken. An diesen Stellen war klinisch eine intensive 
Rötung nachweisbar, mit starker Exfoliation der Epidermis und hier und da fand man anhaftende schuppenartige Krusten, nach deren Loslösung resp. Abtragung oberflächliche Erosionen zum Vorschein kamen.

Ich schnitt des weiteren Hautstïcke von der Bauchwand in der Nähe des Nabels heraus, wo, abgesehen von einer mittelmäßigen Rötung, klinisch keine Veränderungen der Hautoberfläche nachweisbar waren.

Die Stücke wurden mit Alkohol fixiert und in Paraffin und Zelloidin eingebettet and die Schnitte wurden nach den üblichen Verfahren der histologischen Technik gefärbt.

Aus der histologischen Untersuchung ergab sich folgendes:

In den vom Arme, vom Schenkel und vom Rücken entnommenen Stücken ist in erster Linie die starke Verdickung der Epidermis bemerkenswert, welche besonders dle Malpighische Schicht betrifft; besonders stark verdickt sind die interpapillären Ausläufer und dementsprechend sind die Dermapapillen deformiert und reduziert.

Die Verdickung der Malpighischen Schicht ist hauptsächlich durch das parenchymale und interstitielle Ödem ihrer Zellen bedingt. Das interstitielle Ödem überwiegt in der Keimschicht und in den unteren Malpighischen Schichten, während in den oberen ein hydrophischer Zustand der einzelnen Zellen deutlich wird, diese werden groß und rundlich und verlieren ihre Farbenaffinitäten; viele von ihnen weisen die Leloirsche kavitäre Veränderung auf. In den Malpighischen Zellen sind Mitosen sehr selten; es sind keine intraepidermischen Wanderzellen nachweisbar.

Diesen Veränderungen der unteren Epidermissehichten entsprechend, findet man auch solche der Übergangsschichten und der Hornschicht. Das Keratohyalin fehlt im Stratum granulosum, oder ist dort durch einzelne in der Nähe des Kerus gelegene Körnchen kaum angedeutet. Die Kerne sind zusammengeschrumpft und zerstückelt.

Die obere Schicht der Epidermis besteht ans einer mehr oder minder großen, mehrschichtigen und mit stäbchenformigen Kernen versehenen Platte, welche nur in lockerer Weise an den unterliegenden Schichten anhaftet and an vielen Stellen von derselben losgetrennt, frei schwebt (Fig. 1). An einigen Stellen fehlt sie, so daß das Malpighische Netz, deren obere Zellen mehr oder weniger entartet sind, blosgelegt erscheint. Diese Platte stellt vom histologischen Standpunkte die exfoliative Schicht dar.

Diese Veränderungen, welche über große Hautzonen ziemlich gleichmäßig verbreitet sind, erreichen an einigen Stellen vorgeschrittenere Grade. In den oberen Teilen der Malpighischen Schicht ist das parenchymale Ödem stellenweise so beträchtlich, daß die Zelle zerstört wird 
und an ihrer Stelle ein kleiner Hohlraum oder ein rudimentäres Bläschen zurückbleibt, spaltförmig und von den schwer veränderten benachbarten Zellen schlecht abgegrenzt. An anderen Stellen erreichen die Läsionen ein Maximum: der Kolliquationsproze $B$ ergreift die Oberhaut in ihrer ganzen Höhe und der epitheliale Überzug erscheint über eine mehr oder minder lange Strecke unterbrochen, oder, besser gesagt, die gut erhaltene Epidermis der Ränder geht in eine schwer veränderte und fast unkenntliche Epidermis üher, bestehend aus einem kleinmaschigen, Kernfragmente und spärliche Leukozyten enthaltendem Netz (Fig. 2). Dieses den Rest der Epidermis darstellende Netz bedeckt direkt die Dermapapillen. Über demselben breitet sich die Hornschicht aus, welche hier dieselben Charaktere aufweist wie die, welche die Epidermis der Umgebung überzieht.

Das Derma ist in seiner ganzen Höhe vom Hypoderma bis zur Epidermis stark ödematös. Die kollagenen Bündel sind durch breite und unregelmäßige Spalten von einander getrennt. Neben dem Ödem beobachtet man eine enorme Erweiterung der Blutgefäße - besonders der Venen des ganzen Hautnetzes; auch die großen Venen des Hypodermas erscheinen prall und mit Blut gefüllt. Das Ödem und die Gefäßerweiterungen sind ausgesprochener in den Papillen, deren Bindegewebsflechtwerk mit weiten Lymphspalten durchsetzt erscheint. In den Koriumpapillen kann man - nach den üblichen Methoden - kein elastisches Element zum Vorschein bringen; dagegen ist das elastische Gewebe des Stratum reticulare genügend erhalten.

Im Gewebe beobachtet man einen außergewöhnlichen Reichtum an zellularen Elementen. Es handelt sich jedoch größtenteils um Fibroblasten; es sind nur einzelne Lymphozyten nachweisbar. Die Zahl der Mastzellen ist bedeutend vermehrt. Man findet keine Plasmazellen. Ich habe keine Blutergüsse auffinden können.

In den nach Gram behandelten Schnitten findet man in den oberen abblätternden Schichten der Epidermis eine große Menge von Mikroorganismen. Es sind Kokken, die manchmal zu zweien öfters gruppenweise vereinigt sind und zwar wahrscheinlich Staphylokokken, nach ihren Dimensionen und ihrer Gruppierungsweise zu schließen. Dieselben Mikro* organismen findet man an einigen Stellen zwischen den Zellen der Malpighi schen Schicht, anch dort wo diese intakt ist. An den Stellen wo der epidermische Überzug tief verändert ist, beobachtet man auch im Stratum papillare isolierte kleine Kokkengruppen; in einigen Schnitten konnte ich sogar in der subpapillaren Schicht und in dem Coriumflechtwerk Mikroorganismen nachweisen, in den Lymphspalten und zwischen den kollagenen Bündelchen.

Wenn wir nun die hervorragendsten Besonderheiten dieses Falles kurz zusammenfassen, so sehen wir, daß es sich un eine Dermatose handelt, welche ein gesundes Kind, in ausgezeichnetem Ernährungszustande, in den ersten Lebenswoshen befallen hat und zuerst in Form einer Erythrodermie 
aufgetreten ist, welche, von dem Gesichte und genauer gesagt, von der Umgebung des Mundspaltes ausgehend sich rasch ausgebreitet und das ganze Hautgebiet befallen hat. Diese Erythrodermie ist von einer großfetzigen Exfoliation begleitet und gefolgt, welche je nach der Gegend mit dem mehr oder minder starken Auftreten von Feuchtigkeit verbunden ist. Neben der Dermatosis bestehen Magendarmstörungen, welche durch die nekroskopische Untersuchung bestätigt wurden. Das Kind starb infolge von Septikopyämie, wie durch den nekroskopischen und bakteriologischen Befund (Staphylococcus pyogenes aureus) nachgewiesen wurde.

Die histologische Untersuchung der erkrankten Hautabsclnitte hat einen akuten entzündlichen Proze $\mathbf{3}$ mit vorwiegend raskulären und exsudativen Erscheinungen festgestellt, welcher sich im Korium abspielte und dieses in seiner ganzen Höhe interessiert. Die Veränderungen der Epidermis sind sekundär und dem entzündlichen Prozeß des Dermas untergeordnet und haben, obwohl sie einen verschiedenen Grad von Schwere aufweisen, alle dieselbe Bedeutung, indem sie mit der serösen Durchtränkung des Bekleidungsepithels zusammenhängen.

Wenn wir nun zu einer diagnostischen Beurteilung schreiten wollen, so kann meines Erachtens eine Exaltation des erythematösen und exfoliativen Zustandes, welche Neugeborene aufzuweisen pflegen, bei einer eventuellen Differentialdiagnose nicht in Frage kommen, weil dieser nicht entzündliche Zustand eine ganz andere Natur und ein ganz anderes Aussehen hat.

Auch kann es sich in unserem Falle nicht um ein Eczema rubrum handeln, bei welchen, wenn es auch generalisiert ist, der krankhafte Prozeß an einzelnen Stellen sich durch eine feine Bläschenbildung äußert; des weiteren erreicht bei dieser Affektion die Rötung nie eine solche Intensität und schließlich pflegt die Desquamation bei der ekzematösen Dermitis in Form von kleinen, an der Hautobertläche ziemlich antlaftenden Schuppen und nicht in Form von breiten zusammenhängenden Fetzen zu geschehen, wie in unserem Fall.

Der Ton der Rötung und der Turgor der Haut erinnern hei unserem Pat. vielleicht an das Erysipel: es fehlt jedoch 
die hohe Hyperthermie und der scharfe Rand der Läsion, welche zu den Symptomen dieser Hautkrankheit gehören.

Nach Ausschließung dieser Affektionen müssen wir unseren Fall unter die generalisierten Erythrodermien oder besser erythrodermischen Dermatitiden derersten Kindheit klassifizieren. Unter dieser Bezeichnung wird eine sehr unbestimmte und schlecht definierte Gruppe von Dermatosen begriffen, welche zu ganz verschiedenen Deutungen Anlaß gegeben haben.

Unter diesen wollen wir zuerst die Dermatitis exfoliativa neonatorum von Ritter v. Rittershain in Betrachtung ziehen und die klinischen Charaktere derselben genau nach der von Ritter selbst gegebenen Beschreibung lzurz zusammenfassen.

Diese Krankheit befällt fast ausschließlich Neugeborene in der zweiten bis fünften Lebenswoche. Knaben scheinen mehr als Mädchen zu derselben veranlagt zu sein. Die Affektion beginnt mit einer rasch an Intensität zunehmenden Rötung der peribuccalen Gegend; danach bilden sich Rhagaden an den Mundwinkeln. Die Mundschleimhaut ist zuweilen am Prozeß beteiligt. Später breiten sich die Rötung und die Schwellung über die ganze Haut aus. Zuletzt kommt es zum exfoliativen Stadium; die Epidermis schrumpft zusammen und löst sich in breiten Lappen ab und es wird dadurch eine fleischrote Oberfläche freigelegt, auf welcher sich hier und da dünne gelbliche Krusten bilden. In diesem Zustande hat die Haut ein Aussehen wie nach einer Verbrennung. Die Krankheit verläuft fieberlos; in der Hälfte der Fälle hat sie einen tödlichen Ausgang.

Als Ritter von Rittershain 1878 die Dermatitis exfoliativa neonatorum beschrieb, stützte er sich auf die Beobachtung von 297 von ihm während des Dezenniums 1868-1878 im Prager Findelhause untersuchten Fällen. Andere Autoren (Billard, v. Baer, Hervieux, Hütter, Bille) hatten vor Ritter von einem Symptomenkomplex gesprochen, welcher vielleicht der Dermatitis exf. neon. entsprach; Ritter war es aber, der seinen Namen mit dieser Krankheit verknüpfte, indem er die Symptome derselben ausführlich beschrieb, die Anhaltspunkte für eine Differentialdiagnose angab und alles in allem die Krankheit als eine selbständige, von anderen ähnlichen Dermatosen der ersten Kindheit versohiedene morböse Entität hinstellte. Der von Behrend im folgenden Jahre (1879) geäußerten Meinung, daß die Dermatitis exfoliativa mit dem 
Pemphigus foliaceus zu identifizieren sei, erwiderte Ritter v. R. selbst (1880), die Spezifität der Krankheit betonend. Fs folgten die Publikationen von Bohn (1883), Caspary (1884), Elliot (1888), welche einige Fälle von dieser Dermatose mitteilen.

Die Auffassung Ritters erfuhr bei dem $V$. KongreB der Deutschen Gesellschaft für Dermatologie (Graz, 1895) eine weitgehende Bestätigung durch Escherich, welcher 5 Fälle der Krankbeit mitteilte und durch Rosenthal; auch von Kaposi, obwohl dieser in der Deutung der Pathogenese von Ritter v. R. abwich, wurde die Spezifität der Krankheit anerkannt.

Beim nächsten Kongreß, Straßburg 1896, berichteten Caspary, Pick, Rille über einige Fälle von Dermatitis exfoliativa neonatorum, welche sie als eine selbständige, deutlich unterschiedene und wohl definierte morböse Entität ansprachen.

Die anatomisch-pathologischen Untersuchungen von Winternitz (3 Fälle, 1898), Luithlen (2 Fälle, 1899), Bender (3 Fälle, 1900) haben unsere Kenntnisse über die Krankheit bedeutend gefördert. Hansteen hat ebenfalls (1900) 2 Fälle beschrieben und seiner Beschreibung den histologischen Befund beigefügt. Weitere Fälle von Dermatitis exf. neon. wurden schlieflich von Prissmann (1 Fall, 1898), C o m by (4 Fälle, 1898), Kenardath (1 Fall, 1899), Ravogli (1 Fall, 1901), Patek (1 Fall, 1904), Carlton (1 Fall, 1907) mitgeteilt.

Die pathologisch-anatomischen Befunde Winternitz, Luithlen, Hansteen, Bender bei der Dermatitis exf. neon. beziehen sich, obwohl sie sich in einigen Einzelheiten von einander unterscheiden, in der Hauptsache auf ein und dasselbe pathologische Bild.

In den zwei von Hansteen berichteten Fällen hatte der Prozeß eiven äußerst akuten Verlauf, so daß die Krankheit drei Tage nach ihrem Auftreten tödlich endete. Die mikroskopische Untersuchung stellte ein beträchtliches interstitielles und parenchymales Ödem der Epidermis fest; die Hornschicht war von den unterliegenden Schichten losgetrennt; an vielen Stellen erfolgte diese Trennung und Ablösung tiefer zwischen den Zellen des Malpighischen Netzwerkes. Im Korium wurde eine ödematöse Imbibition und eine enorme Dilatation der Blut- und Lymphgefäße und eine spärliche Zellinfiltration nachgewiesen.

Auch Winternitz betont die Bedeutung der Exsulationserscheinungen im Corium, welche die direkte Ursache der Epidermisexfoliation darstellen. Ich fasse die histologischen Veränderungen seines 3. Falles kurz zusammen, dessen Zugehörigkeit zur Dermatitis exf. neon. von $L$ u ithlen anerkannt wurde: In der an einigen Stellen verdickten Epidermis sind hie und da verschiedene Epithelverluste nachweisbar. Die Epidermisexfoliation ist durch das seröse Exsudat des Koriums bedingt, welches durch die Epidermis dringt, sich unter der Hornschicht ansammelt und diese hochlebt. Das Bindegewebe des Koriums ist tief ödematös mit Veränderung des elastisehen Flechtwerkes der Papillar. 
schicht; daneben Blut- und Lymphgefäßdilatation und Blatergüsse in einigen Papillen.

Der Befund des 2. Falles von Bender weist dieselbe Reihe von Erscheinungen, in einem vorgeschritteneren und schwereren Stadium auf. Die Epidermis ist verschwunden oder auf eine dünne, amorphe oder körnige, nicht mehr erkennbare Schicht reduziert. Die Zerstörung erstreckt sich an einigen Stellen auf die Koriumpapillen.

In seinem 3. Falle von Dermatitis exf. neon, hebt Bender hervor, daf eine Lostrennung in der Dicke der Epidermis und genauer in den oberen Schichten des Malpighischen Netzes stattfindet; auch hier wird über eine spärliche Zellinfiltration des Chorions, ödematöse Imbibition des Gewebes und Gefäßerwejterung berichtet.

Luithlen kam bei der histologischen Untersuchung der Haut eines von Dermatitis exfol. neon. befallenen und am 25. Krankheitstage gestorbenen Mädchens zu etwas anderen Resultaten. Dabei ist zu bemerken, daß die Dermatose auf dem Wege der Rückbildung und an einigen Stellen fast verschwunden war, als Pat. infolge eines chronischen Darmkatarrhs starb. Luithlein konnte jedoch Hautstücke des Halses und der Brust, wo die Exfoliation unverändert bestand, histologisch untersuchen. Fr fand eine intensive Wucherung des Malpighischen Netzes in seinen unteren Schichten mit mangelhafter Koratinbildung und Lostrennung der oberen aus kernhaltigen Zellen bestehenden Schichten. Zahlreiche intraepidermatische Leukozyten. In der papillaren und der subpapillaren Schicht bestanden entzündliche Erscheinungen mit einer in der Umgebung der Blutgefäbe besonders intensiven Zelleninfiltration. Nach Luithlens Ansicht stellt die intensive Wuchernng des Malpighischen Netzes den für die Dermatitis exf. neon. charakteristischen Befund dar.

In dem ersten Fall Benders war die Dermatose fast geheilt, als das Kind infolge von Pyämie starb. Der anatomische Befund ähnelte hier demjenigen von $\mathrm{L}$ u i thlen: gesteigerte Wucherung der epidermischen Basalschicht, Verdickung des Netzes, Abnahme des Keratinisierungsprozesses. Bemerkenswert ist die sonderbare Deutung, welche Bender von dem Prozeß gibt: er ist nämlich der Ansicht, daß die Veränderungen der Fipidermis die primären und wesentlichen darstellen, während die entzündlichen Erseheinungen des Koriums sekundär sind und von der Irritation herrühren, welcher dasselbe infolge seines ungenügenden S'chutzes oder seiner Bloßlegung ausgesetzt ist. Diese primären Veränderungen der Epidermis erklärt Bender durch eine hereditäre Anlage und vergleicht die Affektion mit der Epidermolysis bullosa hereditaria (Köbner).

Es ist leicht einzusehen, daß man der Meinung Benders weder vom klinischen noch vom anatomischen Standpunkte aus zustimmen kann.

Den beschriebenen anatomischen Befunden fügen wir nun den unseren hinzu, da wir ohne weiteres den von uns beschriebenen als einen Fall der Krankheit von Ritter ansprechen. 
Diese Befunde weisen übereinstimmend darauf hin, daß eine primäre akute und ausgebreitete Entzündung des Koriums vorliegt mit Gefäßdilatation und ödematöser Exsudation. Die Veränderungen der Epidermis hängen mit der serösen Imbibition des Bekleidungsepithels zusammen und sind, welches anch ihre Intensität sein möge, alle auf das Ödem zurückzuführen. Die Exfoliation (vom histologischen Gesichtspunkte betrachtet) findet in verschiedener Weise statt, je nach der Intensität der Exsudation und dem Stadium des Prozesses: sie kann die oberen, im Zustande der Parakeratose sich befindenden Epidermisschichten betreffen oder die durchdringende Flüssigkeit hebt lie Hornschicht in die Höhe; wenn die Exsudation ausgiebiger und heftig ist, kann die Lostrennung in verschierlenen Höhen der Malpighi schen Schicht stattfinden; schließlich kann man, als den höchsten Grad der Epidermisläsion, die Verschmelzung der Epidermis in toto beobachten.

Die Erythrodermie, welche der Ausdruck des entzündlichen Prozesses im Korium ist, ist und bleibt die fundamentale Erscheinung; die Veränderungen der Epidermis treten, obwohl sie klinisch klarer hervortreten, in den Hintergrund. Wahrscheinlich trägt die Zartheit der Epidermis und das bedeutende Wucherungsvermögen, welches ihre Elemente in dieser Lebensperiode besitzen, bis zu einem gewissen Grade dazu bei, diejenigen Zerstörungs- und Reparationsprozesse zu intensivieren, welche in der Epidermis des Erwachsenen nicht so ausgesprochen sein würden.

Einige Autoren sprechen der Ritter rchen Hautaffektion ihre nosologische Selbständigkeit ab und betrachten sie als eine schwere und ausgebreitete Form des Pemphigus acutus neonatorum: dariiber werden wir uns weiter unten unterhalten.

Hier wollen wir die klinischen und anatomischen Charaktere der von Leiner kürzlich unter dem Namen Erythrodermia desquamativa der Brustkinder beschriebenen Dermatose kurz betrachten. Dieselbe kann unseres Erachtens auf dasselbe pathologische Bild der Ritterschen Dermatose zurückgeführt werden.

Mit dem eben erwähnten Namen hat Le ine $\mathbf{r}$ einen neven Typus von Dermatose des kindlichen Alters beschrieben. Die Krankheit befällt Brustkinder in gutem Ernährungszustande während der ersten drei Lebens- 
monate. Der Verlauf ist fieberlos; es treten oft Durchfälle und Magenstörungen hinzu. Die Dauer des Leidens ist etwas länger als bei der Dermatitis exfoliativa neonatorum.

Im Laufe von 5 Jahren hatte Leiner Gelegenheit, 43 Fälle von Erythrodermia desquamativa zu beobachten, wovon 15 letalen Ausgang hatten. Er betrachtet die Krankheit als eine idiopathische Erythrodermie, ähnlich der Dermatite exfoliatrice idiopathique von VidalB r o c q, welche durch das fettige Aussehen der Schuppen, besonders zu Anfang der Dermatose, durch das Fehlen eines atrophischen Stadiums, und dadurch charakterisiert ist, daß sie aussschließlich Säuglinge in den ersten I ebensmonaten befällt.

Die mikroskopische Untersuchung der erkrankten Haut zeigte einen entzändlichen Prozeb des Koriums, begleitet von einer Erweiterung der Papillenblutgefäße und von einer mittelmäßigen serösen Exsudation, welche sich nie, weder in Räumen der Epidermis, noch in den subepidermatischen Schichten ansammelt, sondern nur eine seröse Durchtränkung des Stratum papillare und der Epidermis bewirkt; Folge davon ist die rasehe Regeneration der Epidermis, die Parakeratose.

Uns scheint auf Grund der Beschreibung von Leiner, daß die Differentialdiagnose zwischen der Erythrodermia desquamativa und der Dermatitis exfoliativa nicht so leicht ist, wie Leiner meint. Beide Hautkrankheiten befallen Kinder in gutem Ernährungszustand und in derselben Lebensperiode; beide verlaufen, wenn keine Komplikationen hinzutreten, fieberlos; auch die Dermatitis exf. ist oft von Magendarmstörungen begleitet (z. B. die Fälle von Winternitz, Comby u. a.). Beide Dermatosen sind klinisch durch eine iiber das ganze Hautgebiet ausgebreitete Erythrodermie und anatomisch durch eine exsudative Phlogose des Choriums und eine seröse Imbibition der Epidermis charakterisiert. Die Exsudation scheint bei der Dermatitis exfoliativa stärker zu sein und die Ablösung von breiten Hautfetzen zu bewirken; die Exfoliation kann sich aber bei der Dermatitis exf. auch nur auf die oberen parakeratotischen Schichten beschränken (siehe den Befund von W in ternitz; auch in unserem Fall, welchen wir auf Grund seines tyischen objektiven Befundes, seines äußerst akuten Verlaufes und der Beteiligung der Schleimhäute am Prozeß als ein Exemplar der Ritterschen Krankheit ansprechen, haben wir bei der histologischen Untersuchung die Exfoliation von parakeratotischen Schichten für weite Strecken gefunden). 
Dafi eine scharfe Trennung der Erythrodermia desquamativa von dem Symptomenkomplex, welcher der Dermatitis exfoliativa entspricht, nicht leicht ist, geht aus der Beschreibung von Leiner selbst hervor, wo dieser Autor auf seine Dermatose die von Luithlen unter dem Namen Dermatitis exf. veröffentlichten Fälle zurückführen will; und wir wollen hinzufügen, daß die Beschreibung der Dermatitis exf. neon., welche Luithlen in Mračeks Handbuch der Hautkrankheiten gegeben hat, viele Berührungspunkte mit der Erythrodermia desquamativa aufweist. Der seborrhoische Charakter der SquamoKrusten auf dem behaarten Kopf und im Gesicht, welcher nach Leiner ein konstanter Charakter der Erythodermia desq. ist, kann meines Erachtens nicht ein entscheidendes Differentialsymptom darstellen, da diese Veränderung bei Säuglingen, und besonders bei solchen, die an Darmkatarrh leiden, bekanntlich äußerst häufig sind. Von dem seborrhoischen Aussehen der Krusten an den erwähnten Stellen ist übrigens auch in den ron Comby berichteten Fällen von Dermatitis exfol. die Rede.

Aus diesen Gründen sind wir der Ansicht, daß die Erythrodermia desq. von Leiner demselben Krankheitsbild der Dermatitis exf. neon. von Ritter v. Rittershain entspricht, und sich von dieser nur durch die längere Dauer, die geringere Schwere und die günstigere Prognose unterscheidet.

Wir glauben des weiteren, daB die von Ritter als Dermatitis exfoliativa neonatorum beschriebene Dermatose eine selbständige morböse Entität darstellt und nicht mit dem Pemphigus acutus contagiosus neonatorum identifiziert werden kann, welcher bekanntlich eine bullöse Dermatose der ersten Kindheit darstellt, die sicher mikrobischen Ursprungs ist, wenn auch die spezielle Natur des Mikroorganismus noch nicht völlig festgestellt ist.

Zur Stütze dieser Deutung führen einige Autoren die Tatsache an, daß die Unterschiede zwischen dem pathologisch anatomischen Befunde des Pemphigus ac. cont. neon. und demjenigen der Dermatitis exf. rein quantitaver Natur sind. Dieser Meinung können wir nicht beitreten. 
Bei diesen beiden Dermatosen findet man in der Tat einen entzündlichen exsudativen Prozeß des Koriums, von welchem die Veränderungen der Epidermis eine direkte Folge sind; der weitere Verlauf der Veränderung beweist aber, daß es sich um zwei deutlich unterschiedene Krankheitstypen handelt. Bei dem Pemphigus ac. cont. neon. befällt das Ödem fast ausschließlich die Papillarschicht und die Gefäßerweiterung ist auf ein umschriebenes Gebiet des oberflächlichen Blutgefäßnetzes lokalisiert: bei dem Pempligus treten die Veränderungen der Epidermis, obwohl sie sekundär sind, vollständig in den Vordergrund, als die charakteristischste und Hauptläsion, und drängen die Koriumläsionen, welche ihre direkte Ursache sind, als Läsionen von geringer Bedeutung in den Hintergrund. Dieses Mißverhältnis zwischen Ursache und Wirkung bei dem akuten Pemphigus der Neugeborenen muß genügend betont werden: ejnem leichtgradigen Ödem des Papillarkörpers entspricht eine beträchtliche intraepidermatische Flüssigkeitsansammlung. Daß die pathologisch-anatomischen Veränderungen des akuten Pemphigus mit demjenigen der Impetigo vulg. verglichen worden sind, ist leicht begreiflich; sowohl bei dieser wie bei jenem kann ron einer Serotaxis die Rede sein, d. h. ron einer Anziehung ron Flüssigkeit aus dem oberflächlichen Chorionblutgefäßnetz nach einem bestimmten, meistens unter der Hornschicht lokalisierten Punkte der Epidermis.

Dagegen ist bei der Dermatitis exf. - von welcher der von uns berichtete Fall ein Beispiel ist - die akute ödematöse Pblogose des Koriums sowohl in Oberfläche wie in Tiefe ausgedehnt: die Dermatitis ist immer die Haupterscheinung und die Veränderungen der Epidermis haben, obwohl sie klinisch deutlicher hervortreten, die Bedeutung einer ausgebreiteten Ernährungsstörung. Der entzündliche Prozeb des Koriums scheint, sozusagen, sich selbst Zweck zu sein: die Läsionen der Epidermis sind eine notwendige Begleiterscheinung, aber von alszesorischer Bedeutung.

Da es sich sowohl bei dem Pemphigus acutus wie bei der Dermatitis exfoliativa um je zwei Prozesse handelt, bei welchen eine Filtration von Exsudatflüssigkeit durch die Epidermis stattfindet, so kommt es nicht selten vor, daß man bei den 
beiden Krankheiten ähnliche Veränderungen der Epidermis beobachtet. So findet man nicht selten in der Nähe der Blasen des Pemphigus acutus Erscheinungen von parenchymatösem und interstitiellem Ödem des Malpighischen Flechtwerkes mit hydropischen Veränderungen der Zellen der Übergangsschichten, und ebenso hier und da eine Wucherung der interpapillären Epidermisausläufer (s. Befund von $\mathrm{Hedinger}$ ).

Bei der Dermatitis exfoliativa kann das durch die Epidermis filtrierende Serum ausgedehnte Loslösungen der Hornschicht, von den unterliegenden Schichten bewirken und es entstehen in dieser Weise Veränderungen resp. Gebilde, welche in klinischer Hinsicht schlaffe, schlecht umgrenzte, an flüssigem Inhalt sehr arme Blasen vortäuschen, somit das klinisehe Bild undeutlicher machen und oft die Ursache diagnostischer Irrtïmer sind.

Jedenfalls genügen diese anatomischen Erscheinungen nicht, um die Individualität der zwei Krankheitsformen in Abrede zu stellen und dürfen nicht als Grund dienen, um diese zu vereinigen und zu verwechseln.

Es handelt sich um zwei Prozesse, welche, obwohl sie sich auf ein und dasselbe Prinzip stützen, in der Entwicklung der Veränderungen in verschiedener Richtung verlaufen und zu verschiedenen Resultaten führen, so dalo sie zwei verschiedene anatomische Entitäten darstellen.

Bezüglich der Differentialdiagnose zwischen der Dermatitis exf. und dem Pemphigus ac. verweisen wir auf die vorangegangenen Publikationen. Hier wollen wir nur einige Betrachtungen einfügen, welche wir der Beschreibung Ritters v. $R$. selbst entnehmen und welche uns zu der Überzeugung führen, daß die ron diesem Autor beobachtete Dermatose nicht als eine schwere und ausgebreitete Form von Pemphigus acutus anzusprechen ist. Obwohl Ritter über ein reichliches Material (fast 300 Fälle) verfügt hat und seine Beobachtungen sich auf 10. Jahre erstrecken, so behauptet er nichtsdestoweniger, daß die Dermatitis exfoliativa nicht kontagiös ist, während bekanntlich der Pemphigus diese Eigenschaft in hohem Grade besitzt. Und wenn man die Dermatitis exfol. als eine Form ron Pemphigus ac. deuten will, $\mathrm{muB}$ es doch auf- 
fallend erscheinen, daß sich diese Dermatitis in einer sogroßen Zahlvon Fällen mitunveränderten Charakteren wiederholthat, ohnedabdazwischen Fällevontypischem Pemphigus acutus aufgetreten sind und die Diagnose klargestellt haben.

Dies erscheint uns merkwürdig auch in Anbetracht einiger veröffentlichten Fälle, in welchen eine Ansteckung gerade die Möglichkeit erbrachte, die vorher zweifelhafte Diagnose klarzustellen. Anderseits werden diese Fälle angeführt, um die Identität der zwei in Frage stehenden Dermatosen zu erweisen.

Fall von Knöpfelmacher und Leiner. Bei einem 5 Tage alten Kind traten zuerst einige schlaffe Blasen auf. Am 11. Krankbeitstage, als K. und L. das Kind wieder untersuchten, war die Haut der Waugen und fast des ganzen Rumpfes intensiv rot und glänzend. Die Epidermis der unteren Extremitäten konnte man in großen Lappen loslösen resp. abziehen. Die Verf. deuten diesen Fall als eine Umwandlung des Pemphigus ac. in Dermatitis exf.

Knöpfelmacher und Leiner beschreiben des weiteren zwei Fälle von Pemphigus acutus, welcher in der Klientel einer Hebamme auftraten, welche auch ein an Dermatitis exfoliativa leidendes Kind in Bèhandlung hatte. Der Verlauf dieses Falles war folgender: Beginn der Krankheit am 5. Lebenstage mit Auftreten einer großen fluktuierenden Blase in der periumbilitralen Gegend. Am nächsten Tage Entstehung: neuer Blasen auf der Thoraxhaut. Danach entwickelte sich das typische Bild der Dermatitis exfoliativa. Das Kind starb am 11. Lebenstage. Ein zweijähriges Schwesterchen, welches in derselben Badewanne gebadet wurde, wurde von einem typischen akuten Pempbigus befallen, welcher heilte. Der anatomische Befund der Fälle von $K$. und $L$. hat eine große Ähnlichkeit mit demjenigen der Dermatitis exfoliativa.

Fall von Hedinger. Gesundes Kind, zur richtigen Zeit geboren. Einige Tage nach der Geburt ausgebreitetes allgemeines Hauterythem. Am 7. Lebenstage erschien eine schlaffe Blase in der Nähe des Nabels; in den folgenden Tagen erschienen ähnliche Blasen auf den Beinen und auf dem linken Daumen. Am 11. Lebenstag, als das Kind in die Klinik aufgenommen wurde, waren die Blasen noch vorhanden; am 12. hatte die. Dermatose ihr klinisches Aussehen geändert und ähnelte demjenigen der Dermatitis exf. Es entstanden keine neuen Blasen: die oberen Epidermisschichten lassen sich auf breiten Zonen über die untenliegenden Schichten verschieben und bochheben, ohne daß unter ihnen eine erhebliche Flüssigkeitsansammlung nachweisbar ist. Wenn man diese Epidermislappen aufhebt, kommt eine stark gerötete, ziemlich trockene Oberfläche zum Vorschein, welche konsistenter ist als die normale Haut. Exitus letalis am 13. Lebenstag (8. Krankheitstag). Der histologische Befund, den ich oben berichtet habe, ähnelt demjenigen der Dermatitis exf. 
Einige Tage später erkrankte ein Kind aus der Kundschaft derselben Hebamme an typischem Pemphigus acutus.

In den eben erwähnten Fällen von Knöpfelmacher und Leiner und ron $\mathrm{H}$ edinger handelt es sich ohne Zweifel um Pemphigus acutus neonatorum, wie aus der anfänglichen. bullösen Eruption und besonders aus der Ansteckung hervorgeht, welche die Entstehung von typischen Pemphigusformen hervorrief. Dann trat aber eine erythematöse Rötung auf, welche sich über gesunde Hautzonen ausbreitete und von einer auf die Dermatitis hinweisenden Anschwellung und später von der Exfoliation begleitet war, und somit das klinische Bild der Ritterschen Dermatose vortäuschte.

Diese Tatsachen zwingen uns aber nicht, den Pemphigus ac. mit der Dermatitis exf. zu identifizieren oder einen Übergang der einen Krankheit in die andere anzunehmen. Wir können mehrere Hypothesen aufstellen: so könnte es sich um bösartige Formen von Pemphigus ac. handeln, mit universaler Ausbreitung und Ablösung von großen Epidermislappen, wie es zuweilen der Fall ist, wenn der Pemphigus Kinder in den ersten Lebenstagen befällt. Man könnte auch an eine Dermatitis denken, welche als eine Komplikation neben der bullösen Initialeruption aufgetreten ist: in diesem Falle wäre jedoch die Dermatitis sekundär, während die Dermatitis exf. immer primär oder idiopathisch ist.

Wie es nun auch sei, diese drei Fälle - welchen man mehrere andere zugesellen könnte - beweisen, daß die Differentialdiagnose zwischen der Dermatitis exf. und dem Pemphigus ac. zuweilen sehr schwierig und fast unmöglich sein kann. Und diese Schwierigkeiten waren jedenfalls nicht die letzte Ursache der Neigung mancher Autoren, die zwei Krankheitsformen zu vereinigen.

Aus einer summarischen Übersicht der Literatur über die Dermatitis exfol. geht hervor, daß einige unter dieser Bezeichnung veröffentlichte. Fälle in Wirklichkeit nicht zu der Ritterschen Dermatose gehören.

So handelt es sich z. B. in dem Fall von D. K. White (1885) nicht um eine Dermatitis exf.: derselbe betrifft ein 10 Tage altes, bei der Geburt vollständig gesundes Kind, welches in einem Zimmer schlief, wo Leuchtgas aus der Leitungsröhre entwich. Zwei Tage später trat ein intensives Erythem auf, und zwar zuerst am Gesicht und dann am ganzen Körper. Dem Erythem folgte eine allgemeine Exfoliation. Das Kind 
wies Symptome von Bronchialirritation und Somnolenz auf. Heilung nach 15 Tagen. Meines Erachtens liegt hier der Gedanke aahe, daß es sich um ein durch das eingeatmete Kohlenoxyd hervorgerufenes toxisches Erythem handelt.

Ich zweifle keinen Augenblick daran, daß der von Walter Spencer (1899) als Dermatitis exf. beschriebenen Epidemie diese Bezeichnung nicht zukommt. Verf. sagt ausdrücklich, daß die initiellen und wesentlichen Effloreszenzen aus ovalen, durchscheinenden, von einem kleinen hyperämischen Hofe umgebenen Blasen bestanden: ein Befund, welcher charakteristisch für den Pemphigus neon. ist. Die starke Desquamation, welche folgte, wenn die Krankheit zur Heilung schriti, hat sich nie über die ganze Körperoberfläche ausgebreitet, und hat nie die Charaktere angenommen, welche der Dermatitis exf. eigen sind.

Auch der von Ravogli (1901) veröffentlichte Fall ist wahrscheinlich als Pemphigus ac. neon. zu deuten.

Bei der Dermatitis exfoliativa neonatorum handelt es sich nach Ritters Ansicht um eine allgemeine Infektion, welche sich fast ausschließlich in der äußeren Haut und an den Übergangsstellen von dieser zu den Schleimhäuten lokalisiert. Escherich ist auch der Meinung, daß diese Dermatose mit einer allgemeinen septischen Infektion zusammenhängt. In diesem Sinne wird sie auch von Rille gedeutet. Pick vergleicht sie mit den septischen Puerperaldermatosen.

Es liegen nur spärliche bakteriologische Untersuchungen über die Dermatitis exf. vor, und alle lassen Raum zu schweren Einwänden.

Winternitz hat in seinem 2. Fall - von welchem Luithlen behauptet, daß es sich um Pemphigus ac. und nicht um Dermatitis exf. handelt - aus dem Blut wiederholt den Staphylococcus pyogenes anreus und albus kultiviert.

Hansteen fand in den mikroskopischen Schnitten seiner zwei Fälle eine große Menge von Staphylokokken, welche sich bis zu den Grenzen zwischen Korium und Epidermis ausbreiteten. Aus der intra vitam entnommenen Flüssigkeit der serösen subepidermatischen Exsudation konnte er den Staphylococcus pyog. aur. züchten.

Ich bin weit entfernt, meinem bakteriologischen Befunde einen für die hier in Frage stehende Dermatose pathogenetischen Wert beizulegen. Der von mir aus dem Herzblut und aus der Milz in Reinkultur gezüchtete und sehr virulente Staphylococcus aureus war höchstwahrscheinlich der Erreger der schweren septikopyämischen Infektion, welche das Kind zum Tode führte. Dieser Staphylococcus entspricht sehr wahrscheinlich den 
gruppenweise vereinigten Kokken, welche ich in den mikroskopischen Schnitten in der freien Epidermisoberfläche and in verschiedener Tiefe in der Malpighischen Schicht gefunden habe. Es könnte aber auch sein, daß dieser Keim der Pathogenese der Dermatose fremd sei und dab er durch die tief veränderte Epidermis sekundär in den Organismus eingedrungen sei.

Wenn man die von Ritter geäußerte Meinung, die Dermatitis exf. habe einen pyämischen Ursprung, auch nicht gänzlich in Abrede stellen kann, so muß man doch wenigstens zugeben, daßj diese Annahme keine Stïtze in der Tatsache findet, daß bei der Dermatitis exf. meistens kein Fieber vorhanden ist und daß die Dermatose, obgleich sehr ausgedehnt, den Allgemeinzustand nicht beeinflußt, ja die Kinder sogar an Gewicht weiter zunehmen.

Deshalb scheint uns die Meinung L e in ersüber die Ätiologie der Erythrodermia sehr annehmbar, wenn er behauptet, es handle sich um ein autotoxisches Erythem, zusammenhängend mit Magendarmstörungen, und wir neigen dazu, diese Auffassung auf die Dermatitis exf. auszudehnen, welche wir aus den angeführten Gründen als mit der Lein erschen Dermatose verwandt betrachten.

Es hatte bereits Luithlen die Möglichkeit erörtert, daß3 die Ätiologie auf toxische Momente zurückzuführen sei; und vom klinischen Standpunkte aus findet das Erythem, mit welchem das Krankheitsbild beginnt, und die rasche allgemeine Verbreitung desselben in dieser Annahme jedenfalls eine befriedigende Erklärung. Die besondere Schwere, welche die Erythrodermie in der Haut des Kindes annimmt, läßt sich durch die strukturellen Eigenschaften der Corium- und Epidermisschichten und besonders durch die besonderen Verhältnisse des Hautlymphkreislaufes (Kroma yer), sowie sie in dieser Lebensperiode sind, in befriedigender Weise erklären.

Wenn wir nun zu einer Schlußfolgerung schreiten wollen, so scheint mir aus meinen Beobachtungen und aus der Besprechung der wichtigsten erschienenen Arbeiten in erster Linie 
hervorzugehen, da $\beta$ die Dermatitis exfoliativa neonatorum von Ritter v. Rittershain eine sowohl in klinischer wie in pathologisch-anatomischer Hinsicht selbständige Krankheit ist. Sie darf deshalb nicht mit dem Pemphigus acutus neonatorum identifiziert werden, obwohl die Differentialdiagnose zwischen diesen beiden Dermatosen zuweilen äußerst schwierig oder unmöglich ist.

Höchstwahrscheinlich gehört die vor kurzem von L e in er als Erythrodermia desquamativa beschriebene Dermatose zu derselben Krankheitsform wie die Dermatitis exfoliativa, von welcher sie sich nur durch die geringere Schwere unterscheidet, und beide Dermatosen sind vom ätiologischen Standpunkte als idiopathische Erythrodermien toxischen Ursprungs anzusprechen.

\section{Literatur.}

v. Ritter. Die exfoliative Dermatitis jüngerer Säuglinge. ZentralZtg. f. Kinderheilkunde. Oktober 1878. (Ref. im Archiv f. Derm. u. Syph. 1879. p. 129.)

Behrend. Vierteljahresschrift f. Derm. u. Syph. 1879. p. 192.

v. Ritter. Die exfoliative Dermatitis jüngerer Sänglinge und Cazenaves Phemphigus foliaceus. (Archiv f. Kinderheilk. I. 2. 1880. Ref. in Vierteljahresschrift f. Derm. u. Syph. 1880. p. 114.)

Behrend. Derselbe Gegenstand. Ebenda. I. 3. 1880.

Bohn. Hautkrankheiten in Gerhardts Handbuch der Kinderkrankheiten.

Ca spary. Vierteljahresschrift f. Derm. u. Syphilis. 1880. p. 122.

Elliot. The americ. Journal of the med. sciences. January. 1888.

White, D. K. Ein Fall von Dermatitis exfoliativa neonatorum. Journal of cutaneous and genito-urinary diseases. August 1895. (Ref. Derm. Zeitschrift Bd. III. p. 223, 1896.)

Verhandlungen der deutsch. derm. Gesellschaft. V. Kongreß zu Graz. 1895. Escherich, Rosenthal. (Diskussion über Pemphigus.)

Verhandlungen der deutsch. derm. Gesellschaft. VI. Kongreß zu Straßburg. 1898. Cas pary: Über Dermatitis exfoliativa universalis. Kaposi, Rille, Arming. (Diskussion.)

Comby J. Dermatite exfoliatrice des nonveaunés in Traité des maladies de l'enfance de Graucher, Comby et Marfan. 1898. p. 279.

Winternitz, R. Ein Beitrag zur Kenntnis der Dermatitis exfoliativa neonatorum (Ritter). Archiv. f. Derm. u. Syph. Festschrift f. Pick. p. 397. 1898. 
Prissmann, S. Ein Fall von Dermatitis exfoliativa neonatorum. St. Petersburg. med. Wochenschrift. 1898. Nr. 50. (Ref. Dermat. Zeitschr. Bd. VI. p. 405. 1899.)

Luithlen, F. Dermatitis exfoliativa (Ritter). Archiv f, Derm. u. Syph. Bd. XLVII. p. 323, 1899.

Spencer, W. An Ontbreak of Dermatitis exfoliativa neonatorum. Austral. Med. Gaz. June, 20. 1899. (Ref. British Journal of Derm. Vol. XI. p. 408. 1899.)

Kenardath, Das. Dermatitis exfol. neonatorum Lancet. 28. Juli, 1899. (Ref. Monatsh. f. prakt. Dermat. Bd. XXX. 1900.)

Hansteen, H. Histologische und bakteriologische Momente zur Ätiologie der Dermatitis exfoliativa neonatorum. Ritter. Archiv f. Derm. u. Syph. Festschrift f. Kaposi. p. 135. 1900.

Bender, 0. Beiträge zur Histologie der Dermatitis exfoliativa nebst einer Bemerkung äber Plasma- und Mastzellen. Virchow s Archiv, Bd. CLIX. p. 86. 1900.

Ravogli A. Ein Fall von Dermatitis exfoliativa neonatorum (Ritter). Cleveland Med. Gaz. August. 1901. (Ref. Derm. Zeitschrift. Bd. IX. p. 401. 1902.)

Richter, P. Über Pemphigus neonatorum. Dermat. Zeitschrift. Bd. VIII. p. 507. 1901.

Luithlen, F. Dermatitis exfoliativa. (Ritter). Mračeks Handbuch d. Hautkrankheiten. Bd. I. p. 755. 1902.

Ostermayer, N. Ein Fall von Pemphigus neonatorum. P. Richter (Dermatitis exfoliativa neonatorum. Ritter) mit Infektion der Mutter und Tod des Neugeborenen. Archiv. f. Derm. u. Syph. Bd. LXVII. p. 110. 1903.

Patek. Dermatitis exfoliativa neonatorum. Ritters disease. Journ. cut. dis. including Syph. June. 1904. (Ref. The british Journ. of derm. Vol. 17. 1905.)

Knöpfelmacher u. Leiner. Dermatitis exfoliativa neonatorum. Jahrbuch f. Kinderheilkunde. Bd. LX. 1904.

Heding er, E. Über den Zusammenhang der Dermatitis exfoliativa neonatorum mit dem Pemphigus acutus neonatorum. Archiv f. Derm. u. Syph. Bd. LXXX. p. 349. 1906.

Carlton, E. P. Dermatitis exfoliativa neonatorum(R i t ter s disease). New-York med. Journ. 28. Sept. 1907. (Ref. Archiv f. Derm. u. Syph. Bd. LXXXIX. p. 462. 1908.

Leiner, O. Über Erythrodermia desquamativa, eine eigenartige universelle Dermatose der Brustkinder. Archiv. f. Derm. u. Syph. Bd. LXXXIX. p. 65. 1908.

\section{Die Erklärung der Abbildungen auf Taf. VI ist dem Texte zu entnehmen.}




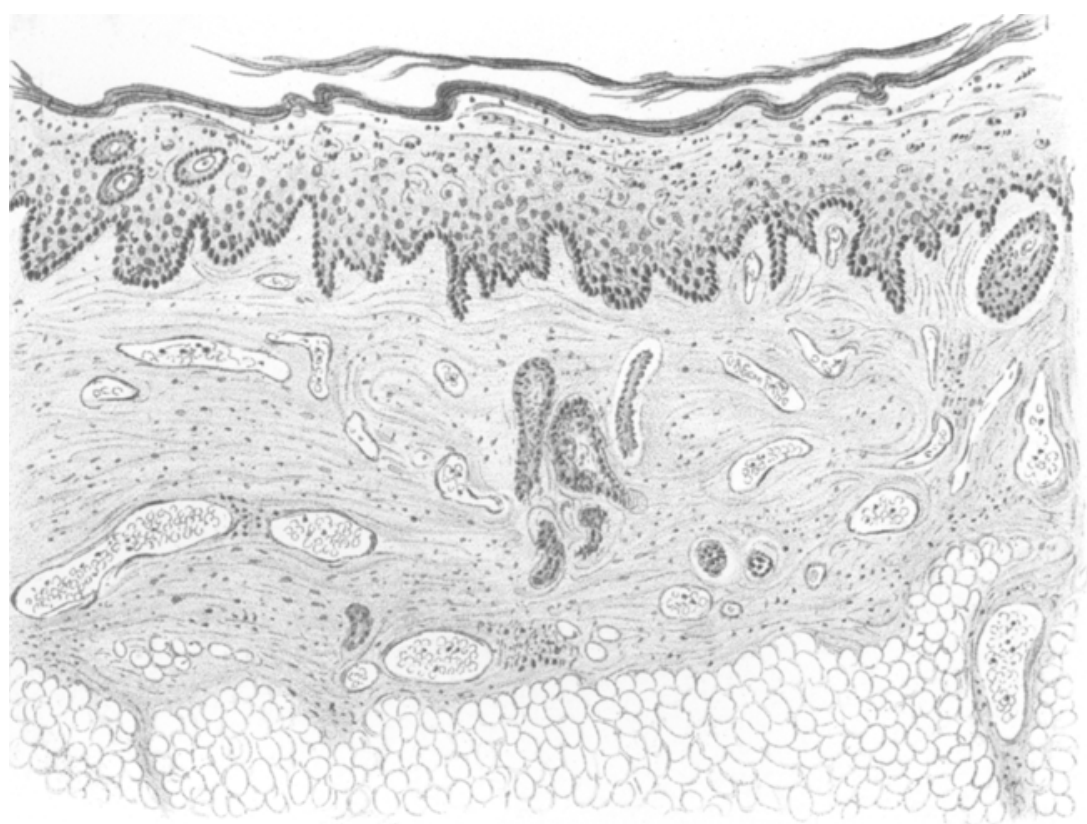

Fug. 1.

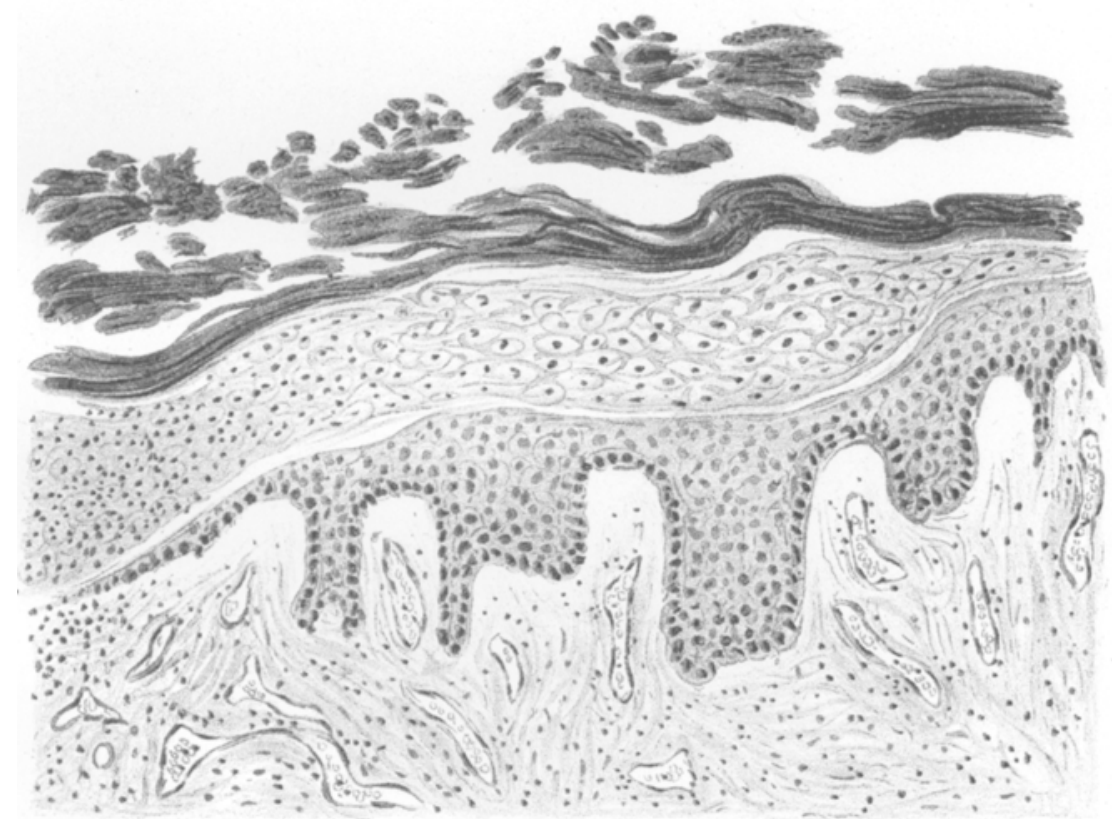

